\title{
Gaucher disease: An orphan disease with significant osseous manifestations
}

\author{
Efstathios Koutsostathis ${ }^{1}$, Konstantinos Tzirogiannis ${ }^{2}$ and Georgios I Panoutsopoulos ${ }^{2 *}$ \\ ${ }^{1}$ Kerameikos Health Center, Athens, Greece \\ ${ }^{2}$ Laboratory of Physiology-Pharmacology, Department of Nursing, Faculty of Human Movement and Quality of Life Sciences, University of Peloponnese, Sparta, Greece
}

\begin{abstract}
Gaucher disease, the most common of the lysosomal storage diseases, is a systematic familial disease classified in "orphan diseases", a group of rare disorders with prevalence of 1:50,000 or lower in the general population. Gaucher disease results from mutations that impair the enzymatic activity of a lysosomal hydrolase called $\beta$-glucocerebrosidase and leads to the accumulation of glucocerebroside, its substrate, in the lysosomes of the macrophage/monocyte system. Macrophages are transformed to Gaucher cells by glucocerebroside accumulation which represent atypical activated macrophages that infiltrate various organs and secrete an array of pro-inflammatory cytokines. This results in organomegally and cytopenias in the peripheral blood due to hypersplenism and infiltration of the bone marrow by Gaucher cells. Cytokines exctreted by Gaucher cells are in the basis of bone pathology. Osteopenia, osteoporosis, painful bone crises, pathologic fractures, and osteonecrosis are the most common manifestations of osseous Gaucher disease. This disease is classified in three types based on CNS involvement and rate of progression. Therapy consists of $\beta$-glucocerebrosidase substitution and substrate reduction therapy.
\end{abstract}

\section{Introduction}

Gaucher disease is the most common of the lysosomal storage diseases, a rare group of approximately 60 inherited metabolic disorders. Today Gaucher disease is classified in "orphan diseases" which comprise a group of rare disorders with prevalence of 1:50,000 or lower in the general population [1]. In 1882, Philippe Gaucher described for the first time a woman with pronounced splenomegaly and unusually large spleen cells and called the disorder "primary spleen epithelioma". The hereditary nature of the disease and the complete spectrum of its systematic manifestations from the internal organs and the central nervous system were fully described in the 20th century. [2].

Lysosomes contain a variety of proteolytic enzymes (hydrolases) as glucosidases, sulphatases, phospholipases, lipases, proteases and nucleases. Genetic defects that impair the enzymatic activity of any of these enzymes lead to the intracellular accumulation of their substrate. For instance, in Pompe disease the accumulating substance is glycogen, in mucopolysaccharidoses the accumulating substance is glucozaminoglycans, and in both types of Nieman-Pick disease as well as in Gaucher disease, Krabbe disease and metachromatic leukodystrophy the accumulating substance is sphingolipids. On other occasions, as in the case of $\mathrm{AB}$ variant of GM gangliosidosis, mutations lead to the impairment of the enzymatic activity of an activator protein or a transporter protein as in the case of Salla Disease and Cystinosis [1,2].

\section{Genetics}

B-glucocerebrosidase is a high molecular weight enzyme consisting of 497 amino acid residues. The GBA1 gene has been mapped in 1q21 chromosomal region where it spans an area of approximately $7,6 \mathrm{~kb}$ [3]. A glucocerebrosidase pseudogene (GBAP) is located $15 \mathrm{~kb}$ downstream of the normal variant. The pseudogene shares $96 \%$ sequence homology with the glucocerebrosidase gene and its biological function remains obscure. The functional normal variant consists of 11 coding areas (exons) and 10 intervening noncoding areas (introns). Today, more than 300 mutations of the GBA1 gene have been identified in patients with Gaucher disease. Most of these mutations represent point mutations that lead to the substitution of a single amino acid, while insertions, deletions, mutations in splice sites and recombination variants with the pseudogene have also been reported. Based on current data, more than $80 \%$ of these mutations are point mutations leading to the substitution of a single amino acid and less than $20 \%$ represent insertions, deletions, frame shift mutations, splice variants and recombinant alleles with the pseudogene. More specifically 4 mutations, N370S, L444P, 84GG, and IVS2, account for the $80 \%$ of the observed phenotypes. N370S, L444P, $\mathrm{D} 409 \mathrm{H}$ are the most prevalent genotypes in the Greek population [4].

Gaucher disease exhibits remarkable phenotypic heterogeneity. N370S allele seems to confer protection against early disease onset while L444P homozygosity is strongly correlated with early disease onset and D409H homozygosity with CNS involvement. Multiple other allele combinations lead to severe impairment of the glucocerebrosidase enzymatic activity and diverse disease phenotypes. Genotype in Gaucher Disease can provide guidance regarding disease severity and progression as well as expected response to treatment. Genotype in Gaucher disease is also pivotal regarding genetic counselling. Despite its prognostic value though, genotype in Gaucher disease cannot by itself lead to definitive prognosis [4]. The disease is more common in specific

${ }^{\star}$ Correspondence to: Georgios I. Panoutsopoulos, Director of Laboratory of Physiology-Pharmacology, Department of Nursing, Faculty of Human Movement and Quality of Life Sciences, University of Peloponnese, Efstathiou \& Stamatikis Valioti and Plateon, Sparta 23100, Greece, E-mail: gpanouts@uop.gr

Key words: Lysosomal storage diseases; Gaucher disease; $\beta$-Glucocerebrosidase; osseous disease

Received: June 18, 2018; Accepted: June 22, 2018; Published: June 30, 2018 
populations as in Ashkenazi Jews and in Northern Sweden (Norbotten area, where a specific subtype of type 3 disease, Norbottnian type, is observed) [1].

\section{Pathophysiology}

Gaucher Disease (GD) results from mutations that lead to impaired enzymatic activity of a lysosomal hydrolase called $\beta$-glucocerebrosidase (GBA1). There are also two other types of $\beta$-glucocerebrosidases, GBA2 and GBA-3 whose biological roles have not been fully elucidated today. All the cells of the human organism are affected by the enzymatic defect and the disorder in inherited as a recessive trait [5].

Glucocerebroside is an intermediate in the metabolism of the glycosphingolipids and is produced from the catabolism of cell membranes especially of cell types with rapid turnover as erythrocytes and leucocytes. Normally glucocerebroside is catabolized to glucose and ceramide by lysosomal GBA1 in the cells of the macrophagephagocyte system. Ceramide is further hydrolyzed to sphingosine by ceramidase.

As with other glycoside hydrolases, the degradation of glucosylceramide by $\beta$-glucocerebrosidase involves acid/base catalysis by two glutamic acid residues and precedes through a two-step mechanism. In the first step, a lysine residue at the active site of the enzyme is being glycosylated using glucocerebroside glucose as substrate and subsequently in the second step glucocerebroside is being hydrolyzed at the $\beta$-site producing glucose and ceramide.

Normally the GBA1 polypeptide synthesized by the ribosomes enters the endoplasmic reticulum (ER). In order to acquire proteolytic activity, the molecule needs to be properly folded through the action of saposins. The saposins are a set of four small glycoproteins located in the endoplasmic reticulum and lysosomes, referred as saposin (Sap) A-D, that act as enzymatic activators in multiple stages of lysosomal sphingolipid degradation, as well as lysosomal membrane digestion. Saposins aid the appropriate folding of the lysosomal and endoplasmic reticulum proteins through interactions with their target-proteins and anionic phospholipids of the inner surface of lysosomal or ER membrane. Activation of GBA1 requires saposin C. After appropriate folding in the ER and acquisition of the necessary for the enzymatic activity tertiary structure, GBA1, as other intracellular proteins, is transferred though vesicles to the Golgi apparatus for further processing including glycosylation [2]. Along with saposins, progranulin is also a molecule of great significance, since its lack leads to accumulation of GBA-1 and loss of its catalytic function [6].

The accumulation of GC in the lysosomes of the macrophages of the affected individuals has severe metabolic consequences, since they are transformed to activated macrophages, not capable to carry out their normal functions. The macrophages of patients with Gaucher disease (Gaucher cells) infiltrate spleen, liver and the bone marrow and rarely can give rise to tumor like growths in internal organs called "Gaucherosomes". In addition, it is significant to stress the fact that glucocerebroside is not accumulated in the lysosomes of osteoclasts and osteoblasts. In electron microscopy Gaucher cells are full of giant lysosomes with characteristic crinkles or striations. The activated macrophages secrete a wide spectrum of cytokines, which are in the basis of pathophysiology and osseous manifestations of the disease. These cytokines include, among others, TNFa, IL-6, IL-1p, IL-10, Serum Angiotensin-converting enzyme and cathepsin K [7]. Two types of activated macrophages are implicated in Gaucher disease: Type 1 Gaucher cells -also called the classical ones- promote type 1 activation of T-Helper lymphocytes inducing chronic inflammation. Type 2 Gaucher cells have antiinflammatory action, producing and array of antiinflammatory cytokines such as IL-10, IL1Ra [8]. The ratio of CD4/CD8 cells is also reduced in the disease. Gaucher cells retain their phagocytic properties against pathogens but they are defective in red cell phagocytosis due to reduced respiratory burst and ROS production as well as impaired chemotaxis. That is why many partially degraded red cells are found in the bone marrow of the affected individuals. The total net effect of the above is the establishment of a chronic inflammatory phenotype, which also leads to significant changes in the intracellular $\mathrm{Ca}++$ concentrations [7-10].

Chitotriosidase is a chitinase (glucosaminidase) enzyme of the human plasma. Its activity is greatly elevated, approximately 1000 times the upper limit, in Gaucher disease representing a significant marker for the diagnosis and follow up of the disease. Gaucher cells express monocytic markers as CD14, CD68, CD163 and MHC II antigens [8].

Ceramide insufficiency has also significant implications. Ceramide is considered an antioncogenic factor. It blocks the transforming signal of the p38 dependent MAP/ERK kinases and its reduction explains partially the increased incidence of plasmatocytoma and other neoplasias as hepatocellular carcinoma in Gaucher Disease [11].

\section{Clinical manifestations}

Disease severity varies greatly from the invariably mortal infantile type (II) at one end to the completely asymptomatic type at the other. In general, all types of Gaucher disease are characterized by some degree of internal organ and osseous involvement with overlapping clinical manifestations. According to the International Collaborative Gaucher Group (ICGG) at the Gaucher Registry, clinical manifestations of the disease include the following: splenomegaly $85 \%$, thrombocytopenia (with or without hemorrhage) 68\%, hepatomegaly 63\%, osteopenia $55 \%$, growth retardation $36 \%$, anemia $34 \%$, bone pain $33 \%$, fractures $7 \%$, bone crises $7 \%$.

Gaucher disease is classified in 3 major clinical types, delineated by the absence or presence of neurologic involvement and progression:

- Type 1 - Non-neuronopathic Gaucher disease, is characterized by the absence of CNS involvement and accounts for the 95\% of cases in patients of Caucasian origin.

- Type 2 - Acute neuronopathic Gaucher disease, manifests early at infancy and is characterized by intense CNS involvement with life expectancy less than two years.

- Type 3 - Chronic neuronopathic Gaucher disease, is characterized by less severe CNS involvement, splanchnic involvement and life expectancy that reaches adulthood [1-3,12].

Recent recognition of neurological component in type I disease as Parkinson Disease and peripheral neuropathies has led to the conclusion that disease clinical phenotype should be considered as a continuum and not as discrete clinical subtypes [13].

Type 1 is the most common type in the Western World (1:40,000100,000 in the general non Jewish population). Age of onset and disease progression vary greatly even in patients homozygous for the common mutation N370S (whose phenotype is considered non severe) as well as in siblings. The major characteristics of type 1 are pancytopenia, hepatosplenomegaly and osseous disease. In general, phenotypic heterogeneity seems to correlate with the genotype although differences in the clinical expression of the disease are observed 
in identical homozygous twins. Most patients have unexplained splenomegaly at the time of diagnosis with or without anemia and thrombocytopenia. Osseous disease might be absent or severe with osteonecrosis and spontaneous fractures. Symptomatic splenomegaly and thrombocytopenia are the most common clinical manifestations and so the majority of patients are investigated initially in hematology clinics. Sometimes thrombocytopenia and splenomegaly might be mild and the diagnosis is accidentally set. Splenomegaly might be massive with spleen size reaching 20 times the upper normal limits and impacting upper abdomen. Massive splenomegaly is also associated with splenic infarcts. Hepatomegaly is also another classical finding, which is accompanied by ischemia or fibrosis in $20 \%$ of patients. Leucopenia is also common, increasing the risk of infection. Cytopenias in Gaucher disease are usually caused by hypersplenism and to a lesser degree by bone marrow infiltration by Gaucher cells [1-3].

\section{Pathogenicity of osseous Gaucher disease}

Osteoclasts regulate the function of osteoblasts, the release of blood cells from the bone marrow and they also act as immune cells during inflammatory states.

There are two main ways in which Gaucher disease results in osseous disease. First, the accumulation of glucocerebroside in the macrophage cells leads to expansion of the bone marrow, and the red pulp of the spleen. This leads to alterations of the microanatomy of the bones and vessel engorgement which finally may result to vascular occlusion and splenic infarcts and osteonecrosis. Second, the activated macrophages excrete cytokines that stimulate bone absorption, either by activating osteoclasts or by inhibiting osteblasts. These cytokines are: IL-1B, IL-6, M-CSF,TNF-a and other proteins like MIP-1a or MIP$1 \mathrm{~b}[9,12]$.

Beside cytokines, the activated macrophages secrete hydrolases like TRAP (Resistant to trygid acid oxine phosphatase). TRAP is stimulated by cathepsin $\mathrm{K}$ and its activation leads to reduced bone density [14].

Collectively, osseous involvement in Gaucher Disease is described by the model of the "osteoimmunological background of the disease" [15].

\section{Clinical manifestations of osseous Gaucher disease}

The clinical manifestations of osseous Gaucher Disease can take any form of bone disease. Osteopenia, osteoporosis, osteonecrosis, painful bone crises, bone growth retardation are all observed in patients with the disease as well as the characteristic radiographic Erlenmeyer flask sign.

\section{Osteopenia/Osteoporosis}

Both osteopenia and osteoporosis refer to abnormal reduction of bone mass and density. Both cortical and medullary bone are affected. Bone resorption in Gaucher disease results from increased osteoclast activity and can be diffuse or localized [16]. Reduction of bone density classically correlates with increased incidence of abnormal bone fractures. Additionally, the bone mass index is significantly low. By the age of 12-20 years the phenomenon of osteopenia escalates [17].

\section{Osteonecrosis (avascular necrosis)}

Osteonecrosis constitutes the most serious clinical manifestation from the bones in Gaucher disease. Osteonecrosis develops as a result of chronic ischemia. Osteonecrosis is accompanied by severe pain and reduced mobility of the affected bones, pathologic fractures and destruction of the implicated joints. Surgical intervention is often required for restoration of joint function and motility.
Osteonecrosis can influence both cortical and medullary bone and osteonecrosis of the medullary bone is characteristically asymptomatic. Head of the femoral bone is the most common site of osteonecrosis and many patients experience multiple episodes. In children, it can manifest as Legg-Calve-Perthes disease [1].

\section{Painful bone crises}

Painful bone crises manifest with local tenderness and inflammation of the affected bones and fever. They often lead to severe mobility impairment and patients can be bedridden for many days and sometimes for weeks. Increased serum inflammatory markers and leukocytosis are also occasionally observed [16].

The differential between painful bone crisis and osteomyelitis can be troublesome, since the clinical manifestations are almost identical. Patients with Gaucher disease are susceptible to infections, especially from Gram-positive bacteria, due to the nonfunctional macrophagesGaucher cells. The best test for discriminating these clinical entities is PET-scan which reveals "hot spots" in osteomyelitis and "cold spots" in painful bone crisis of Gaucher Disease [18].

\section{Pathologic fractures}

They are observed in patients with osteopenia, painful bone crises and osteonecrosis. The fractures may happen practically everywhere at the skeleton. In a survey of 9 patients over 25 years with a total of 23 fractures, half of them were located in bones where a bone crisis had happened in a period of 2 months to 1 year earlier [19].

\section{Growth retardation}

Growth retardation is often accompanied by inability to reach maximal bone density and is not accompanied by pain. Half of the pediatric patients with Gaucher disease were at the lowest 5th height percentile for their age, in one survey. This condition is attributed to a phenomenon called "resting energy expenditure". However, there are surveys that suggest that children with the disease finally reach a normal height at adulthood. It should also be mentioned that Gaucher disease is not correlated with dwarfism [20].

\section{Erlenmeyer flask sign}

It is caused by severe impairment of normal bone remodeling and aberrant regulation of bone absorption and mineralization. It is present in $60 \%$ of patients. It manifests as deformity of the long bones usually the femoral bones especially at their distal end and at the proximal end of the tibia. Histologically, it is characterized by expansion of the metaphyseal areas due to impaired remodeling and osteoclastic activity. The metaphyseal regions look like a "sparkling flame". Besides problematic remodeling, osteoclast dysfunction at the Ranvier ring, the layer of the cortical metaphyseal bone adjacent to the epiphysis of the bone, is also implicated [16].

There many surveys that suggest that splenectomy, for the management of splenomegaly and hypersplenism, increases significantly skeletal pathology, probably due to the accumulation of glucocerebrosides in the mitochondria of the bone cells since spleen, the major site of their deposition, is removed [13].

\section{Gaucher disease and dental disease}

All long bones can be affected by the disease. Mandibular involvement has been described in at least 100 cases, with the diagnosis to be set accidentally by plain X-Rays for other reasons. Significant osteopenia, loss of the trabecular architecture of the bone, along with 
pseudocysts or honeycomb osseous abnormalities were observed in all cases [21]. A case of periodontitis in a patient with Gaucher disease has also been reported [22]. Radiological findings from the teeth include, generalized osteopenia, osteosclerosis and enlargement of medullary spaces [23].

\section{Uncommon clinical manifestations}

Besides common clinical manifestations other rarer symptoms and signs might be present in some patients such as primary or secondary hypertension, face and leg melachrosis, liver cirrhosis and portal hypertension (usually in splenectomized patients), hepatorenal syndrome, calcification of cardiac valves (in type 3c), renal involvement with nephrotic syndrome, recurrent bacterial infections, isolated vertebral compression fractures, iliopsoas hematomas, and tumor-like Gaucher cell deposits called "gaucheromas» as well as mesenteric lymphadenopathy. Incidence of gallstones is increased in Gaucher disease. Hepatomegally is a common finding while evolution to true liver cirrhosis and failure are much rarer clinical manifestations. Cirrhosis is rarely accompanied by portal hypertension, esophageal varices and ascites with the exception of splenectomized patients [24,25].

Severe lung disease with alveolar and interstitial infiltration by Gaucher cells is exceptionally rare and can also be accompanied by vascular infiltration, pulmonary fibrosis and restrictive lung disease. Although rare, this clinical entity has been described with great detail due to its severity and grief prognosis [26].

\section{Gaucher disease and neoplasia}

Increased incidence of blood malignancies has been reported in Gaucher disease. Very often Gaucher disease is connected with immune system abnormalities as polyclonal hyperglobulinemia which is present at $14-41 \%$ of adult patients at the time of diagnosis. Long term antigenic stimulation may lead to chromosomal instability and multiple myeloma through activation of histidine deaminases [27]. Incidence of premalignant conditions is also increased mainly monoclonal gammopathy of unknown significance (MGUS) which is observed in $25 \%$ of patients while multiple myeloma is increased 5,9 to 51,1 times in patients with the disease in relation to the general population. This is also the case for other malignancies as Non-Hodgkin lymphomas and primary amyloidosis. Additionally, hepatocellular carcinoma is 100 times more common in patients with Gaucher disease in relation to the general population. Cholangiocarcinoma is also more prevalent in Gaucher disease where it is accompanied by increased secretion of glucosylsphingosin and cholesterol bile stones [16,28].

\section{Diagnosis of Gaucher disease}

Early diagnosis of the disease is crucial since most patients have significant splanchnic involvement at the time of diagnosis (in types 1 and 3). The major diagnostic criterion is reduced enzymatic activity of $\beta$-glucocerebrosidase. Genetic testing of the gene sequence allows genotyping. Bone marrow biopsy should not be performed given that the procedure is invasive, has low specificity and consequently high false positive rates [29].

The majority of patients with Gaucher disease exhibit clinical signs of chronic inflammation with occasionally mild multifocal lymphadenopathy and histological findings in biopsy specimens compatible with nonspecific reactive hyperplastic lymphadenopathy. Laboratory findings are also indicative of chronic inflammation with reduced albumin levels, increased ESR and increased serum acute phase reactants and pro-inflammatory cytokine levels as indicated by increased serum CRP. Not rarely, monoclonal zones are also present that can actually represent or evolve to plasma cell dyscrasias. Incidence of an autoimmune disease and neoplasia are also increased based on the findings of large cohort studies with predominantly increased risk for lymphohyperplastic diseases of B cell origin [30].

Definitive diagnosis of the disease requires the measurement of the enzymatic activity of $\beta$-glucocerebrosidase in white blood cells or fibroblasts. Genetic testing/molecular analysis is also required for definitive diagnosis. Plasma chitotriosidase levels are increased due to its increased production from Gaucher cells. Residual $\beta$-glucocerebrosidase enzymatic activity does not correlate with disease severity.

In type 1 of the disease, the diagnosis is set by the clinical manifestations and the relevant laboratory findings in combination with the past medical history and family history of the patient. Typical findings include organomegaly, increased transaminase levels, thrombocytopenia or pancytopenia. Gaucher cells are detected in all tissues of the afflicted individuals. Measurement of $\beta$-glucocerebrosidase enzymatic activity in peripheral blood leucocytes or in fibroblasts after skin biopsy sets the diagnosis, which is confirmed with genetic testing [15]. Recently, a newer simplified method using dried peripheral blood drops has been developed for the measurement of $\beta$-glucocerebrosidase enzymatic activity. Chitotriosidase levels can also be measured as they provide an index of response to treatment and consequently can be used for patient follow up [31].

Chemokine CC (CCL18/PARC) is also measured since its levels are elevated 10-40 times above normal in Gaucher disease and fall with treatment. Its measurement is especially useful when chitotriosidase levels are inconclusive. Ferritin, immunoglobulin and angiotensin converting enzyme levels (ACE) as well as acid phosphatase levels (TRAP) are also measured [32].

Complete blood count, liver function tests, BUN, creatinine, coagulation panel and serum iron levels are also necessary tests for the diagnosis and monitoring response to treatment. High immunoglobulin levels, low cholesterol levels and PT/APTT prolongation are observed in $30 \%$ of patients [15].

\section{Imaging in Gaucher disease}

Skeletal involvement is considered a sign of grave prognosis since it can lead to serious complications with significant morbidity and mortality. Imaging modalities in Gaucher disease includes X-rays, MRI, DEXA scan for bone density measurement and bone scans. $\mathrm{X}$-rays are mainly used for the imaging of bone cortex and can detect lytic or sclerotic lesions as well as fractures. X-rays also detect intraosseous bowing and the Erlenmeyer flask sign but they cannot detect lesions in the bone marrow cavity.

Osteopenia in Gaucher disease is generalized and is usually visible at advanced stages. The most accurate method for bone density measurement is DEXA scan and accurate measurement requires exclusion of osteonecrotic areas.

Bone marrow is best visualized with MRI and yellow bone marrow is best studied on sequences T1-W1 and T2. Increased signal in sequence $\mathrm{T} 2$ is considered indicative of edema and inflammation, as in painful bone crisis or infection.

More contemporary techniques as MRS mass spectrometry and bone scan with Tc $99 \mathrm{~m}$ as well as bone scan with labeled white 
blood cells are useful in pediatric patients that cannot be subjected to X-rays and in the differential diagnosis of painful bone crisis from osteomyelitis. Numerous scoring systems have been proposed for disease staging with $\mathrm{BMB}$ score to be the most commonly used. BMB score incorporates signal density and topography in conventional MRI sections in the lumbar spine and femoral bones [12,33].

\section{Radiographic findings}

- The most common sites of bone involvement are the skull and the head and the shaft of the femur.

- The major pathognomonic radiographic finding is Erlenmeyer flask sign.

- Vertebral bodies, head of the humerus and the pelvic bones can also be afflicted.

- Vertebral body collapse leads to compression fractures and sometimes compression of the spinal cord.

- In bone marrow Gaucher cells invade and dislocate normal hematopoietic cells.

- Osteopenia can also lead to pathologic fractures.

- Infiltration of the bones by Gaucher cells causes lytic lesions that sometimes resemble bone metastasis requiring biopsy for definitive diagnosis [16].

\section{Treatment}

Clinical diversity, varying progression and high treatment cost all necessitate individualized treatment of patients with Gaucher disease.

In nonneuronopathic type 1, two of the following manifestations must be present for initiation of treatment: hepatic, splenic, cardiac, pulmonary or renal involvement, anemia, platelet count below 120.000/ $\mathrm{ml}$, painful bone crisis or active bone disease (osteopenia, bone infarcts, osteonecrosis, lytic bone lesions, fracture), height at the lower 2.5th percentile for age in children and family history of the disease [15].

Before 1990, disease treatment was only symptomatic since no specific etiologic treatment had developed. Symptomatic treatment included correction of anemia with transfusions, bone surgery or arthroplasty for osteonecrosis, and pathologic fractures and general supportive treatment. Splenectomy was performed in patients with hypersplenism albeit increasing the risk of infection, osteonecrosis and pulmonary hypertension [24].

The first successful etiologic treatment with allogeneic bone marrow transplantation was performed in 1980. Enzymatic substitution began in 1991 with alglucerase and three years later imiglucerase, a recombinant enzyme was available. In 2003, a taskforce group published detailed guidelines and therapeutical goals for Gaucher disease. Individualized treatment is emphasized in these guidelines.

The three main $\beta$-glucocerebrosidase substitution therapies developed until today use mannose receptor as intracellular carrier and are: 1. Imiglucerase, 2. Velaglucerase and 3. Taliglucerase.

Despite their efficacy, the major side effect of all is antigenicity, which poses serious problems [15,34]. Substrate reduction therapy (miglustat) may be used in adult patients with moderate disease. These medications inhibit UDP-glucose-ceramide-glucosyltransferase, the first enzyme in the enzymatic path of sphingolipid glycosylation. None of the above therapies is effective against the neuronopathic type of the disease [35].
The therapeutic targets include: 1 . Normal CBC without the need for blood transfusions, since the anemia in Gaucher disease may be extremely severe, 2 . Reduction of liver (20\%-30\%) and spleen mass (30\%-50\%) over 2 years and 3. Elimination, of the skeletal manifestations of the disease including restoration of normal bone growth.

Follow up is of extremely important and is performed on a biannual basis. It includes CBC, LFTs, $\beta$-glucocerebrosidase, and chitotriosidase levels. MRI of the abdomen is also necessary in cases with massive hepatosplenomegaly $[34,35]$. Early detection of hematologic and other malignancies is also a major goal as well as early recognition of type 3 signs and symptoms (eg eye movement abnormalities). Finally, correlation of Gaucher disease with iron deficiency anemia and insulin resistance should be kept in mind [34,35].

\section{Perspectives- conclusions}

The issues and challenges regarding Gaucher disease are multiple. The most important of them are described below:

1. Deciphering the vast phenotypic heterogeneity in a single gene disease. This is important for prenatal testing, especially in types 1 and 3 .

2. Understanding the systematic nature of the disease and multi-organ involvement

3. Important questions regarding splenic infarcts and osteonecrosis remain to be resolved.

4. Understanding the effects of the disease on macrophages. The role of platelets and endothelial cells should also be elucidated.

5. Evaluation of the efficacy of available treatments.

6. Identification of new biomarkers that could guide therapeutic decisions.

7. Reduction of treatment cost.

8. Elucidation of the mechanism of correlation with Parkinson disease. For instance, why are persons with only one mutation in an allele of GBA1 gene at increased risk for developing parkinsonism?

9. Understanding of the mechanism mediating neuronal insult in the neuronopathic type of the disease. What is the explanation for the great variety and complexity of related phenotypes?

10.Development of effective treatments for the neuronopathic types of the disease.

11. Understanding disease characteristics in other areas outside the Western Hemisphere as Asia and Africa where there is lack of data [36].

\section{Declaration of conflicting interests}

The authors declared no potential conflicts of interest with respect to the research, authorship, and/or publication of this article.

\section{Funding}

The authors received no financial support for the research, authorship, and/or publication of this article.

\section{References}

1. Stirnemann J, Belmatoug N, Camou F, Serratrice C, Froissart R, et al. (2017) A review of Gaucher disease pathophysiology, clinical presentation and treatments. Int $\mathrm{J} \mathrm{Mol} \mathrm{Sci}$ 18: E441. [Crossref] 
2. Ferreira CR, Gahl WA (2017) Lysosomal storage diseases. Transl Sci Rare Dis 2: 1-71. [Crossref]

3. Rosenbloom BE, Weinreb NJ (2013) Gaucher disease: a comprehensive review. Crit Rev Oncog 18: 163-175. [Crossref]

4. Human gene database (http://www.hgmd.cf.au.uk/ac/index.php)

5. Yildiz Y, Hoffmann P, Vom Dahl S, Breiden B, Sandhoff R, et al. (2013) Functional and genetic characterization of the non-lysosomal glucosylceramidase 2 as a modifier for Gaucher disease. Orphnet J Rare Dis 8: 151. [Crossref]

6. Mistry PK, Liu J, Sun L, Chuang WL, Yuen T, et al. (2014) Glucocerebrosidase 2 gene deletion rescues type 1 Gaucher disease. Proc Natl Acad Sci USA 111: 4934-4939. [Crossref]

7. Panicker LM, Miller D, Awad O, Bose V, Lun Y, et al. (2014) Gaucher iPSC-derived macrophages produce elevated levels of inflammatory mediators and serve as a new platform for therapeutic development. Stem Cells 32: 2338-2349. [Crossref]

8. Sheth JJ, Sheth FJ, Oza NJ, Gambhir PS, Dave UP, et al. (2010) Plasma chitotriosidase activity in children with lysosomal storage disorders. Indian J Pediatr 77: 203-205. [Crossref]

9. Allen MJ, Myer BJ, Khokher AM, Rushton N, Cox TM (1997) Pro-inflammatory cytokines and the pathogenesis of Gaucher's disease: increased release of interleukin-6 and interleukin-10. QJM 90: 19-25. [Crossref]

10. Mizukami H, Mi Y, Wada R, Kono M, Yamashita T, et al. (2002) Systemic inflammation in glucocerebrosidase-deficient mice with minimal glucosylceramide storage. J Clin Invest 109: 1215-1221. [Crossref]

11. Kitatani K, Wada M, Perry D, Usui T, Sun Y, et al. (2015) Activation of p38 Mitogenactivated protein kinase in Gaucher's disease. PLoS One 10: e0136633. [Crossref]

12. Grabowski GA, Petsko GA, Kolodny C (2001) Gaucher Disease. The online metabolic and molecular basis of inherited diseases, NY: Mc GrawHill.

13. Linari S, Castaman G (2015) Clinical manifestations and management of Gaucher disease. Clin Cases Miner Bone Metab 12: 157-164. [Crossref]

14. Lacerda L, Arosa FA, Lacerda R, Cabeda J, Porto G, et al. (1999) T cell numbers relate to bone involvement in Gaucher disease. Blood Cells Mol Dis 25: 130-138. [Crossref]

15. Zafeiriou D, Marinakis T, Simeonidis A (2017) Gaucher disease: clinical, pathophysiological and therapeutic approach, Publisher: Medical Society of Athens, $43^{\text {rd }}$ Panhellenic Medical Congress, 38-42.

16. Elstein D, Itzchaki M, Mankin HJ (1997) 11 Skeletal involvement in Gaucher's disease. Baillières Clin Haematol 10: 793-816.

17. Mistry PK, Weinreb NJ, Kaplan P, Cole JA, Gwosdow AR (2011) Osteopenia in Gaucher disease develops early in life: Response to imiglucerase enzyme therapy in children, adolescents and adults. Blood Cells Mol Dis 46: 66-72. [Crossref]

18. Bell RS, Mankin HJ, Doppelt SH (1986) Osteomyelitis in Gauche disease. J Bone Joint Surg Am 68: 1380-1388. [Crossref]

19. Katz K, Cohen IJ, Ziv N, Grunebaum M, Zaizov R, et al. (1987) Fractures in children who have Gaucher disease. J Bone Joint Surg Am 69: 1361-1370. [Crossref]
20. Kauli R, Zaizov R, Lazar L, Pertzelan A, Laron Z, et al. (2000) Delayed growth and puberty in patients with Gaucher disease type 1: natural history and effect of splenectomy and/or enzyme replacement therapy. Isr Med Assoc J 2: 158-163. [Crossref]

21. Bender IB (1938) Dental observations in Gaucher's disease. J Dent Res 17: 359-369.

22. Horwitz J, Hirsh I, Machtei EE (2007) Oral aspects of Gaucher's disease: a literature review and case report. $J$ Periodontol 78: 783-788. [Crossref]

23. Nobre RM, Ribeiro AL, Alves-Junior SM, Tuji FM, Rodrigues Pinheiro Md, et al. (2012) Dentomaxillofacial manifestations of Gaucher's disease: preliminary clinical and radiographic findings. Dentomaxillofac Radiol 41: 541-547. [Crossref]

24. Freund MR, Reissman P, Zimran A, Alberton J (2018) Splenectomy in Gaucher disease: a call for minimally invasive surgery. Surg Laparosc Endosc Percutan Tech 28: 86-89. [Crossref]

25. Lachman RH, Wight DG, Lomas DJ, Fisher NC, Schofield JP, et al. (2000) Massive hepatic fibrosis in Gaucher's disease: clinicopathological and radiological features. OJM 93: 237-244. [Crossref]

26. Mistry PK, Sirrs S, Chan A, Pritzker MR, Duffy TP, et al. (2002) Pulmonary hypertension in type I Gaucher's disease: genetic and epigenetic determinants of phenotype and response to therapy. Mol Genet Metabol 77: 91-98. [Crossref]

27. Taddei TH, Kacena KA, Yang M, Yang R, Malhotra A, et al. (2009) The underrecognized progressive nature of N370S Gaucher disease and assessment of cancer risk in 403 patients. Am J Hematol 84: 208-214. [Crossref]

28. Mistry PF, Sadan S, Yang R, Yee J, Yang M (2007) Consequences of diagnostic delays in type 1 Gaucher disease: the need for greater awareness among hematologistsoncologists and an opportunity for early diagnosis and intervention. Am J Hematol 82: 697-701. [Crossref]

29. Stone WL, Master SR (2018) Gaucher disease. Stat Pearls Publishing.

30. Rogowski O, Shapira I, Zimran A, Zeltser D, Elstein D, et al. (2005) Automated system to detect low-grade underlying inflammatory profile: Gaucher disease as a model. Blood Cells Mol Dis 34: 26-29. [Crossref]

31. Lei K, Zhao Y, Sun L, Liang H, Luo R, et al. (2018) A Pilot screening of high-risk Gaucher disease children using dried blood spot methods in Shandong province of China. Orphanet J Rare Dis 13: 48-24. [Crossref]

32. Boot RG, Verhoek M, de Fost M, Hollak CE, Maas M, et al. (2004) Marked elevation of the chemokine CCL18/PARC in Gaucher disease: a novel surrogate marker for assessing therapeutic intervention. Blood 103: 33-39. [Crossref]

33. Rosenthal DI, Scott JA, Barranger J, Mankin HJ, Sani S, et al. (1986) Evaluation of Gaucher disease using magnetic resonance imaging. J Joint Surg Am 68: 802-808. [Crossref]

34. Bennett LL, Fellner C (2018) Pharmacotherapy of Gaucher disease: current and future options. PT 43: 274-309. [Crossref]

35. Hughes D, Cappelini MD, Berger M, Van Droogenbroeck J, de Fost M, et al. (2007) Recommendations for the management of the haematological and onco-haematological aspects of Gaucher disease. Br J Haematol 138: 676-686. [Crossref]

36. Mistry PK, Lopez G, Schiffmann R, Barton NW, Weinreb NJ, et al. (2017) Gauche disease: Progress and ongoing challenges. Mol Genet Metab 120: 8-21. [Crossref]

Copyright: (C)2018 Koutsostathis E. This is an open-access article distributed under the terms of the Creative Commons Attribution License, which permits unrestricted use, distribution, and reproduction in any medium, provided the original author and source are credited. 